\title{
Accumulation of VEGFR2 in zoledronic acid-treated endothelial cells
}

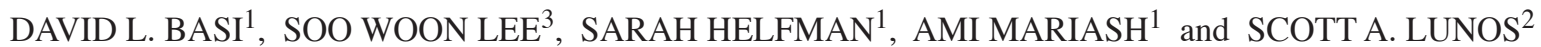 \\ ${ }^{1}$ Division of Oral and Maxillofacial Surgery, University of Minnesota Dental School, Minneapolis, MN 55455; \\ ${ }^{2}$ Biostatistical Design and Analysis Center, Clinical and Translational Science Institute, University of Minnesota, \\ Minneapolis, MN 55414, USA; ${ }^{3}$ Busan Paik Hospital, Inje University, Busan 614-735, Korea
}

Received December 31, 2009; Accepted March 8, 2010

DOI: $10.3892 / \mathrm{mmr}+00000271$

\begin{abstract}
Nitrogen-containing bisphosphonates (BIS) are potent inhibitors of bone resorption and are used in the treatment of a number of medical conditions, including multiple myeloma, breast cancer and osteoporosis. Recent experimental evidence demonstrates that BIS also affect endothelial cell functions and angiogenesis; however, the molecular mechanism(s) are unclear. Vascular endothelial growth factor (VEGF) is a potent pro-angiogenic signal for endothelial cells. BIS inhibit VEGF responses in endothelial cells. The VEGF receptor-2 (VEGFR2) is the main signaling receptor for VEGF in endothelial cells. We hypothesized that altered VEGFR2 expression in BIS-treated endothelial cells may account for these attenuated responses to VEGF. The affect of the BIS zoledronic acid (ZOL) was investigated in human umbilical vein endothelial cells using confocal microscopy, Western blotting, real-time PCR and flow cytometry. VEGFR2 accumulated within the ZOL-treated endothelial cells $(p=0.0002)$, though not on the cell surface $(p>0.05)$. ZOL did not induce VEGFR2-specific mRNA $(p>0.05)$. ZOL inhibited endothelial cell chemotaxis towards VEGF ( $p=0.001)$. VEGF stimulation significantly reduced the amount of VEGFR2 in the endothelial cells $(\mathrm{p}=0.01)$. This response to VEGF was reduced by ZOL ( $p>0.05)$. The effects of $\mathrm{ZOL}$ on endothelial cell migration, VEGFR2 protein expression and response to VEGF were attenuated by geranylgeranyl pyrophosphate. Two- and one-way ANOVAs with Tukey or Dunnett's multiple comparison adjustments were used. The data suggest that ZOL induces aberrant VEGFR2 accumulation. This is not likely due to the induction of mRNA transcription, but rather to the disruption of the mevalonate pathway.
\end{abstract}

Correspondence to: Dr David L. Basi, Department of Developmental and Surgical Science, Division of Oral and Maxillofacial Surgery, University of Minnesota Dental School, 515 Delaware Street SE, 7-174 Moos Tower, Minneapolis, MN 55455, USA

E-mail: basix001@umn.edu

Key words: pathogenesis, endothelial cells, bisphosphonates, vascular endothelial cell growth factor receptor-2

\section{Introduction}

Nitrogen-containing bisphosphonates (BIS) are potent inhibitors of bone resorption and are used during the treatment of many diseases, including multiple myeloma and metastatic breast cancer $(1,2)$. Recent in vitro and in vivo studies have suggested that BIS also affect endothelial cell functions and angiogenesis. The BIS zoledronic acid (ZOL) inhibits cytokine-stimulated endothelial cell proliferation and migration (3), suggesting that ZOL inhibits cellular responses to cytokines. Furthermore, BIS affect angiogenesis in vivo. ZOL inhibits testosterone-stimulated angiogenesis within the prostate in mice (4). In an experimental cervical cancer model, ZOL increased endothelial cell apoptosis and subsequently reduced angiogenesis (5). BIS also altered vascular response to wound healing. ZOL reduced the number of endothelial cells within alveolar bone after tooth extraction (6). Collectively, these findings suggest that ZOL is capable of inhibiting endothelial cell function and angiogenesis. The molecular mechanisms accounting for the aberrant responses to growth factors and cytokines caused by BIS remain largely unknown.

VEGF interacts with many receptors, including VEGFR1/ fms-like tyrosine kinase (FLT)-1 and VEGFR2/KDR/ fetal liver kinase (FLK)-1. Experimental evidence supports VEGFR2 as the major receptor mediating cell signaling in endothelial cells in response to VEGF. Selective activation of VEGFR1 does not induce cell migration or intercellular calcium release in endothelial cells (7); furthermore, transgenic mice with VEGFR1 lacking the cytoplasmic portion had apparently normal vasculogenesis and angiogenesis (8). In vitro, VEGF stimulation induces minimal tyrosine VEGFR1 phosphorylation in endothelial cells (9). By contrast, the functional inactivation of VEGFR2 by a blocking antibody disrupted angiogenesis (10). Endothelial cells expressed increased VEGFR2 mRNA at the site of arterial injury (11). VEGF-stimulated endothelial cells phosphorylate VEGFR2 and downstream signaling targets, such as PLC- $\gamma$ (12). These findings suggest that VEGFR2 is the main receptor responsible for signal transduction during VEGF-stimulation in endothelial cells. Therefore, we hypothesized that BIS modulate VEGFR2 expression in endothelial cells. 


\section{Materials and methods}

Cell culture. Human umbilical vein endothelial cells (HUVECs) were isolated, characterized and grown as previously described $(13,14)$.

Cell migration and quantitation. HUVECs (75,000 cells/well) were placed onto 24-well transwell tissue culture plates with an $8-\mu \mathrm{m}$ pore size (Corning Inc., Life Science). Cells were incubated with or without ZOL (ChemPacific), or with or without geranylgeranyl pyrophosphate (GGPP) (Sigma-Aldrich Corp.) for $48 \mathrm{~h}$ at $37^{\circ} \mathrm{C}$. Next, the cells were incubated in serum-free media for $8 \mathrm{~h}$ prior to the addition of VEGF (R\&D Systems) to several of the lower transwell chambers. HUVECs were allowed to migrate for $16 \mathrm{~h}$ at $37^{\circ} \mathrm{C}$. The undersides of the transwell inserts were washed, and the remaining cells were fixed with $5 \%$ formalin. Next, the cells were stained with hematoxylin and mounted on glass slides. Cells from each treatment group were counted with the aid of a light microscope.

Confocal microscopy, image management and quantification. HUVECs were rinsed with ice-cold PBS and fixed in freshly prepared $4 \%$ paraformaldehyde for $10 \mathrm{~min}$. After cell fixation, the cells were washed with PBS ( $3 \times 5$ min) and then incubated with PBS containing $0.1 \%$ saponin. Cells were blocked and permeabilized with $0.1 \%$ bovine serum albumin BSA in PBS plus $0.1 \%$ saponin for $30 \mathrm{~min}$ at RT. In all subsequent staining steps, the PBS included $0.1 \%$ saponin. Next, the cells were incubated with VEGFR2 antibodies (R\&D Systems) according to the manufacturer's recommended dilution for at least $1 \mathrm{~h}$. The wells were washed ( $3 \times 5 \mathrm{~min})$ with PBS containing $0.1 \%$ saponin, and incubated with Alexafluorlabeled isotype-specific secondary antibodies (Molecular Probes). Excess secondary antibodies were removed by washing. Fluorescent images were visualized with a Bio-Rad MRC 1024 Confocal Laserhead on an Olympus AX-70 upright microscope, x60 water immersion lens, using Bio-Rad Laser Sharp 3.0 Software with Confocal Assistant. Each confocal image was colorized in a standard fashion using Adobe Photoshop CS software (Adobe Inc.). Quantification of fluorescence was performed according to the methods developed at the University of Minnesota Biomedical Image Processing Laboratory (15). Cell fluorescence was distinguished from the background with the 'threshold' tool, and the cells of interest were circumscribed with the 'lasso' tool. The number of cells and positive pixels (fluorescence) from each individual image were calculated (number of red pixels/cell).

Semi-quantitative real-time PCR. Total RNA was isolated from HUVEC monolayers by the guanidium-phenol extraction method with TRIzol reagent (Invitrogen) according to the manufacturer's protocol. The total amount of RNA isolated from each sample was quantified by absorbance at $260 \mathrm{~nm}$. Equal amounts of total RNA (800 ng) were converted to cDNA using an Advantage RT reverse transcription kit (Clontech) according to the manufacturer's protocol. The cDNA from each treatment group was analyzed for VEGFR2 using VEGFR2-specific primers (5'-TCA CTT TGT GCA AGA TAC CCA GA-3'). The reaction mixture consisted of Taq SYBR Green Supermix with Rox (Bio-Rad) in a $25-\mu \mathrm{l}$

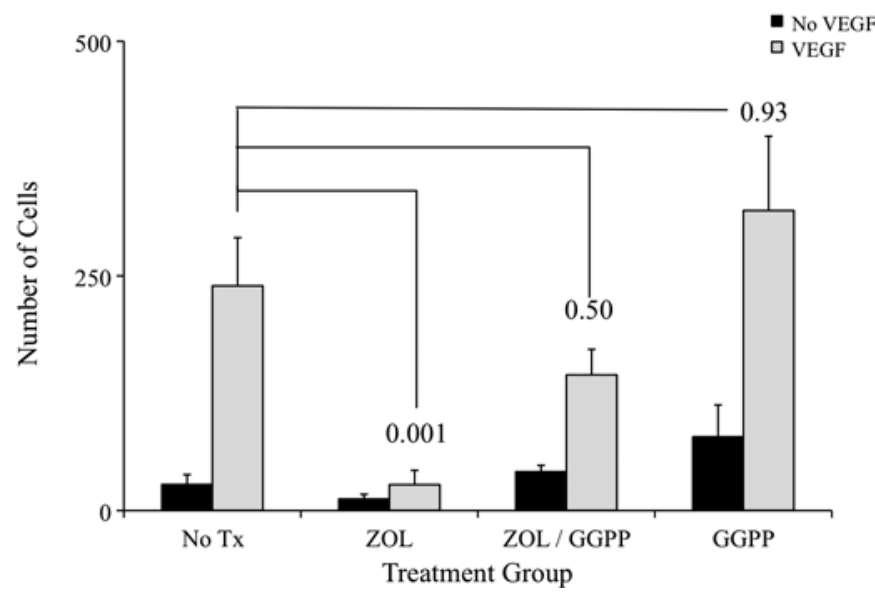

Figure 1. ZOL inhibits endothelial cell migration towards VEGF. Equal number of HUVECs were incubated with or without $12.5 \mu \mathrm{M}$ ZOL and/or $3 \mu \mathrm{M}$ GGPP in transwell cell culture plates. After $48 \mathrm{~h}, 25 \mathrm{ng} / \mathrm{ml}$ of VEGF (grey bars) or cell culture media without serum (black bars) was added to the lower chamber. The cells were incubated for $16 \mathrm{~h}$ at $37^{\circ} \mathrm{C}$. Next, the cells that migrated through the porous membrane were washed, fixed, stained and counted. Bars represent the mean \pm standard error $(n=5)$.

total reaction mixture. Amplification was performed by 30-35 cycles consisting of denaturation at $95^{\circ} \mathrm{C}$ for $30 \mathrm{sec}$, primer annealing at $62^{\circ} \mathrm{C}$ for $60 \mathrm{sec}$ and primer extension at $72^{\circ} \mathrm{C}$ for $30 \mathrm{sec}$ on a Thermocycler (Stratagene Model Mx3000p).

Western blotting. Proteins from treated HUVEC grown on $100-\mathrm{mm}$ plates were isolated with RIPA buffer. An equal amount of protein from HUVEC lysates was separated by 6\% SDS-polyacrylamide gel electrophoresis. Separated proteins were transferred to Hybond ECL paper (Amersham Bioscience), blocked with 5\% milk and then incubated with VEGFR 2 or human $\beta$-actin (Sigma-Aldrich Corp.) antibodies. After washing, blots were incubated with HRP-conjugated secondary antibodies (Santa Cruz). Excess secondary antibody was removed by washing. The blots were incubated with Supersignal (Pierce) to react with the remaining HRP-conjugated secondary antibodies. Chemiluminescence was detected by radiographic film. The radiographic films were digitized and appropriate sized bands were analyzed by densitometry using NIH ImageJ software (version 1.42).

Flow cytometry. HUVECs were analyzed for surface immunoreactive VEGFR2 protein (iVEGFR2) expression by flow cytometry. HUVEC monolayers were dispersed by incubation with $0.5 \mathrm{mM}$ EDTA/0.017\% trypsin as previously described (14). Dispersed HUVECs were washed with cold PBS containing $10 \%(\mathrm{v} / \mathrm{v})$ FBS, pelleted and then counted in a hemacytometer. Equal numbers of HUVECs were resuspended in PBS containing 2\% (v/v) FBS and incubated with a VEGFR2 antibody (per the manufacturer's recommended concentration; R\&D Systems) or an IgG isotype-specific control antibody. Next, excess primary antibodies were removed by washing with PBS containing $2 \%$ FBS, and the cells were then incubated with an FITC-labeled anti-isotype secondary antibody (Jackson ImmunoResearch Laboratories). Excess secondary antibodies were removed by washing and 
A
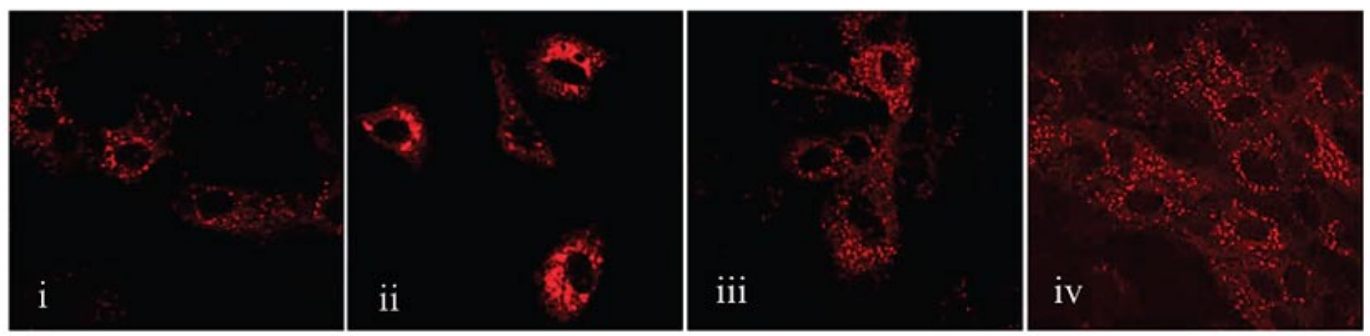

B

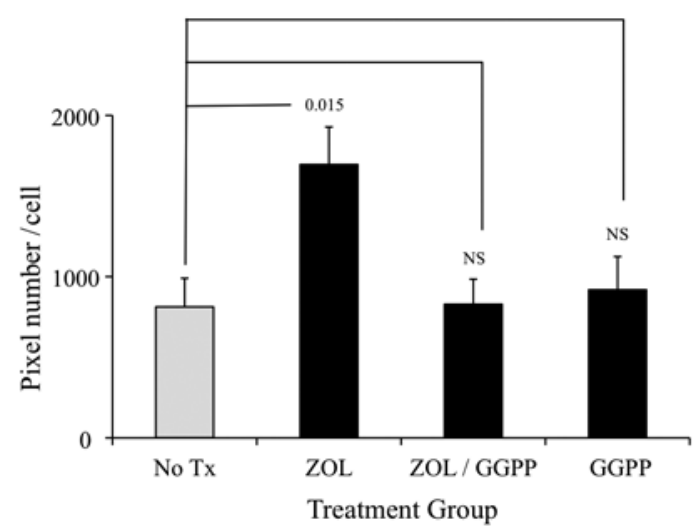

C

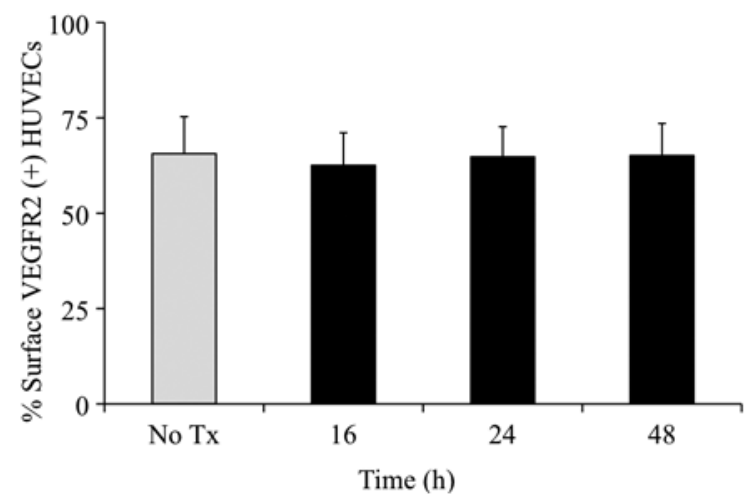

Figure 2. VEGFR2 accumulation within ZOL-treated HUVECs. (A) Representative confocal microcopy images of VEGFR2-stained HUVECs. HUVECs were incubated with (ii) or without (i) $12.5 \mu \mathrm{M}$ ZOL; some cell cultures contained $12.5 \mu \mathrm{M}$ ZOL and $3 \mu \mathrm{M}$ GGPP (iii), and others contained only $3 \mu \mathrm{M}$ GGPP (iv). Cells were stained for VEGFR2 (red) and then viewed by confocal microscopy. Images were digitized and the number of red pixels per cell was determined using computer software. (B) Accumulated confocal microscopy data. Confluent HUVECs were treated with $12.5 \mu \mathrm{M}$ ZOL for various time periods. Surface VEGFR2 was analyzed using flow cytometry (C). Results are reported as the mean \pm standard error (confocal microscopy, $n=6$; flow cytometry, n=9). NS, not significant.

the cells were assessed for surface VEGFR2 by detecting the amount of fluorescence per cell using a flow cytometer (Beckton Dickinson FACScan).

Statistical methods. Analysis of variance (ANOVA) was used to test for differences among the means of multiple treatment groups. When the overall test revealed the results to be significant (at a p-value of 0.05), pairwise comparisons were made using Tukey's multiple comparison tests or Dunnett's test, when comparing treatment groups to the 'no' treatment group.

\section{Results}

ZOL inhibits endothelial cell chemotaxis towards VEGF, which is prevented by GGPP. To determine whether ZOL alters endothelial cell migration towards VEGF, equal numbers of HUVECs from the different treatment groups were allowed to incubate for $48 \mathrm{~h}$ prior to chemotaxic induction. The HUVECs were then allowed to migrate towards serum-free cell culture medium containing VEGF for $16 \mathrm{~h}$. ZOL significantly reduced endothelial cell migration towards VEGF (Fig. 1). Since BIS is a known inhibitor of farnesyl pyrophosphate synthase (16), by preventing the biosynthesis of GGPP we aimed to determine whether the addition of GGPP prevents the effect of ZOL on HUVEC migration. We therefore also incubated endothelial cells with ZOL and GGPP for $48 \mathrm{~h}$, then allowed the HUVECs to migrate towards VEGF. GGPP prevented the effect of ZOL on endothelial cell chemotaxis (Fig. 1). HUVEC cell migration to VEGF was not significantly altered by GGPP treatment alone.
ZOL causes VEGFR2 to accumulate within endothelial cells. To determine whether VEGFR2 protein expression is altered by ZOL, HUVECs were incubated with ZOL for $48 \mathrm{~h}$, then the presence of the protein was visualized by immunofluorescence. iVEGFR2 was initially evaluated by confocal microscopy. Endothelial cells expressed constitutive amounts of iVEGFR2, which significantly increased after ZOL incubation (Fig. 2A, i vs. ii, and B). GGPP prevented the increase in iVEGFR2 expression caused by ZOL (Fig. 2A, i vs. iii, and B). GGPP alone did not significantly alter iVEGFR2 expression (Fig. 2A, i vs. iv, and B). Next, to determine whether the VEGFR2 protein also accumulated on the endothelial cell surface, we analyzed surface iVEGFR2 by flow cytometry. The number of iVEGFR2(+) HUVECs was not significantly altered by $\mathrm{ZOL}$ at the time points tested (Fig. 2C).

VEGFR2-specific mRNA expression is not altered in ZOL endothelial cells. To determine whether VEGFR2 mRNA transcription paralleled the increased VEGFR2 protein expression, HUVECs were incubated with ZOL for various time periods. At each time point, cells were harvested and the total RNA was isolated and analyzed for VEGFR2specific mRNA by real-time PCR. ZOL treatment did not significantly alter VEGFR2 mRNA expression at the time points tested (Fig. 3A). To confirm and extend our confocal microscopy data, iVEGFR2 expression was determined in the ZOL-treated HUVECs by Western blotting. The mean amount of iVEGFR2 increased and became statistically significant after $32 \mathrm{~h}$ of ZOL treatment compared to the non-treated cells (Fig. 3B). 
A

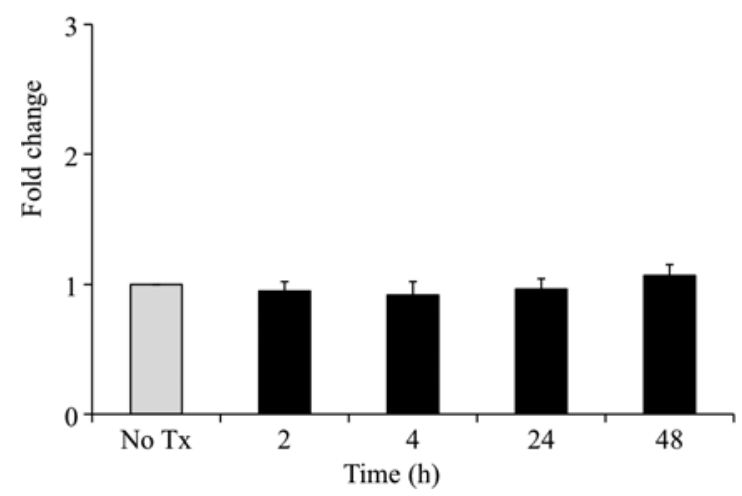

B

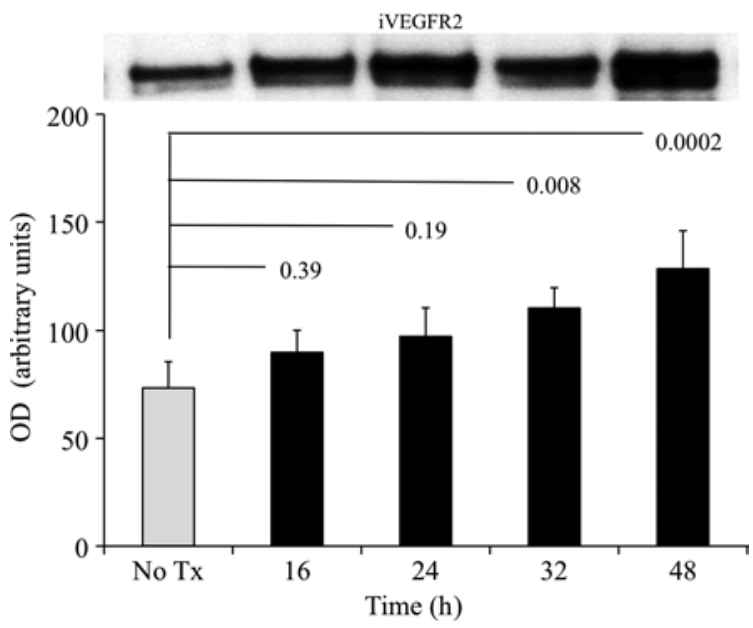

Figure 3. ZOL does not induce VEGFR2-specific mRNA in HUVECs. Confluent HUVECs were treated with $12.5 \mu \mathrm{M}$ ZOL for various times. Total RNA, protein or cells were isolated from each treatment group and analyzed for (A) VEGFR2 mRNA by RT-PCR or (B) whole cell protein expression by Western blotting. (B) An image of a representative blot is shown above the accumulated data. Bars represent the mean \pm standard error (RT-PCR, $n=3$; Western blotting, $n=6$ ).

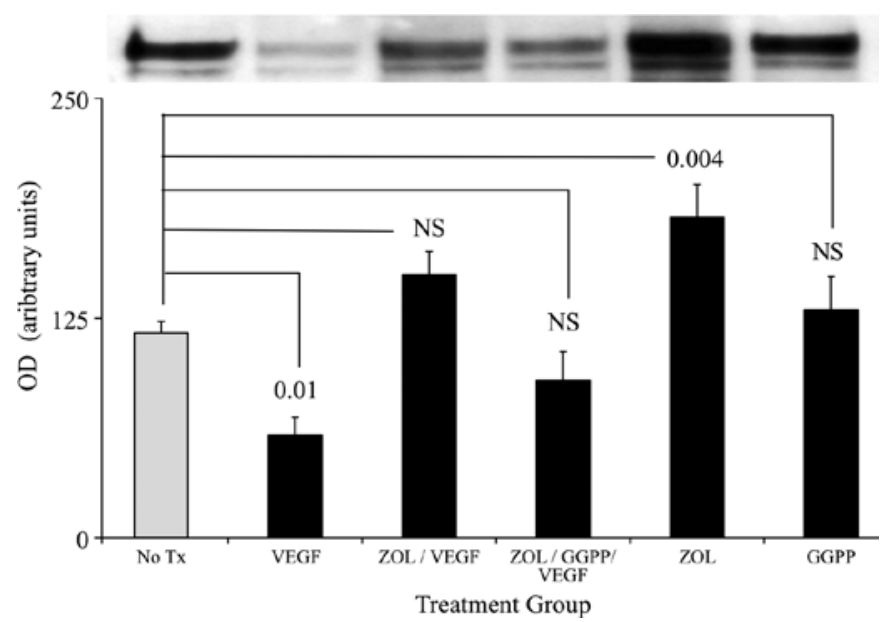

Figure 4. ZOL reduces the amount of VEGFR2 degradation in VEGFstimulated HUVECs. HUVECs were incubated with or without $12.5 \mu \mathrm{M}$ ZOL. Additionally, selected cell cultures were also incubated with $3 \mu \mathrm{l}$ GGPP. After the initial treatment with ZOL and/or GGPP, certain cell cultures were stimulated with $10 \mathrm{ng} / \mathrm{ml}$ VEGF. Cellular protein extracts were analyzed for iVEGFR2 by Western blotting. The blot lanes above the bar graph correspond to the $\mathrm{x}$-axis labels of the accumulated optical density data. Bars represent the mean \pm standard error; $n=4$. NS, not significant.

VEGFR2 is not altered after VEGF stimulation in ZOL-treated endothelial cells. To determine whether VEGFR2 protein levels are modulated after receptor activation, HUVECs were treated with or without ZOL and then certain cell cultures were stimulated with VEGF for $6 \mathrm{~h}$. VEGF stimulation caused a significant reduction of iVEGFR2 in endothelial cells (Fig. 4). The amount of iVEGFR2 was significantly greater in ZOL-treated HUVECs (no Tx vs. ZOL). VEGF stimulation of ZOL-treated HUVECs reduced the amount of iVEGFR2 (compare no Tx vs. ZOL/VEGF to no Tx vs. ZOL). After VEGF stimulation (VEGF vs. ZOL/VEGF, p=0.002) a significantly increased amount of iVEGFR2 remained in the ZOL-treated HUVECs. GGPP attenuated the effects of ZOL on VEGFR2 expression after VEGF stimulation (VEGF vs. ZOL/GGPP/VEGF, p=0.37). HUVEC iVEGFR2 expression was not significantly altered by GGPP (no Tx vs. GGPP).

\section{Discussion}

BIS disrupt endothelial cell function and angiogenesis in vitro and in vivo (17). The molecular mechanisms that underpin these aberrant responses to cytokines, such as VEGF, are not well understood. We hypothesized that BIS alter VEGFR2 expression in endothelial cells, which may lead to abnormal responses to VEGF. To confirm that BIS attenuate endothelial response to VEGF, a chemotaxis assay was performed. ZOL significantly reduced endothelial cell migration towards VEGF (Fig. 1). BIS is a known inhibitor of farnesyl pyrophosphate synthase (16), and consequently prevents the biosynthesis of geranylgeranyl pyrophosphate (GGPP). We aimed to determine whether this pathway is involved in the attenuated endothelial response to VEGF. The results of the present study show that the addition of GGPP abated abnormal endothelial cell migration (Fig. 1) and the accumulation of VEGFR2 (Figs. 2 and 3) caused by ZOL, suggesting that the inhibition of the farnesyl pyrophosphate synthase is, in part, responsible for the reduced endothelial cell chemotaxis towards VEGF.

To determine whether VEGFR2 protein expression is altered by ZOL, endothelial cells treated with ZOL were analyzed by two methods: confocal microscopy (Fig. 2) and Western blotting (Fig. 3B). ZOL caused a significant increase in VEGFR2 protein accumulation in endothelial cells, which was prevented by concomitant GGPP treatment (Fig. 2), again suggesting that the disruption of the malvanoate pathway is at least partially responsible for the effects observed by ZOL. VEGFR2 protein appeared to accumulate with increased duration of exposure to ZOL (Fig. 3B), yet the surface expression of VEGFR2 was not apparently affected by ZOL treatment (Fig. 2C), suggesting that VEGFR2 accumulated within the endothelial cells. To determine whether ZOL induced de novo VEGFR2-specific mRNA, the relative mRNAs were evaluated using real-time PCR. We found that the mRNA from ZOL-treated endothelial cells did not cause a comparable increase in VEGFR2 transcription (Fig. 3A), suggesting that the observed increase in VEGFR2 protein expression was due to post-translational stabilization of VEGFR2 protein and/or reduced VEGFR2 degradation. 
A portion of the total amount of VEGFR2 protein is degraded after VEGF stimulation (18). To determine whether VEGFR2 is degraded after VEGF stimulation in ZOL-treated endothelial cells, endothelial cell cultures were incubated with VEGF after ZOL treatment. VEGF stimulation significantly reduced the amount of VEGFR2 compared to non-treated cells (Fig. 4), suggesting that a portion of the VEGFR2 was degraded. ZOL significantly affected the amount of VEGFR2 protein that remained after VEGF stimulation (Fig. 4; VEGF vs. ZOL/VEGFR treatment groups, $\mathrm{p}=0.002$ ). However, stimulation with VEGF reduced the amount of VEGFR2 in the ZOL-treated endothelial cells compared to non-treated cells (Fig 4; compare no Tx vs. ZOL/VEGF to no Tx vs. ZOL). However, the reduction in the percentage of the VEGFR2 was less in the ZOL-treated endothelial cells after VEGF stimulation (percentage reduction in the mean $\mathrm{OD}, 49 \%$ reduction compared to no Tx vs. VEGF, and $18 \%$ reduction in $\mathrm{ZOL}$ vs. ZOL/VEGF). Furthermore, the quantity of VEGFR2 was statistically similar when comparing the ZOL/VEGF and ZOL treatment groups $(p=0.27)$. The data imply that VEGF interacts with VEGFR2 and may cause lysosomal degradation of the receptor, but this is hindered by $\mathrm{ZOL}$.

In conclusion, we demonstrated that VEGFR2 accumulates within ZOL-treated cells, which may be reduced by GGPP, suggesting that the attenuated response to VEGF involves disruption of the mevalonate pathway and the VEGFR2.

\section{Acknowledgements}

We thank the Department of Medicine at the University of Minnesota, and specifically Ms. Julia Nguyen for the isolation and characterization of the HUVECs used in our study.

\section{References}

1. Carteni G, Bordonaro R, Giotta F, Lorusso V, Scalone S, Vinaccia V, Rondena R and Amadori D: Efficacy and safety of zoledronic acid in patients with breast cancer metastatic to bone: a multicenter clinical trial. Oncologist 11: 841-848, 2006.

2. Lipton A, Small E, Saad F, Gleason D, Gordon D, Smith M, Rosen L, Kowalski MO, Reitsma D and Seaman J: The new bisphosphonate, Zometa (zoledronic acid), decreases skeletal complications in both osteolytic and osteoblastic lesions: a comparison to pamidronate. Cancer Invest 20 (Suppl 2): 45-54, 2002.

3. Wood J, Bonjean K, Ruetz S, Bellahcene A, Devy L, Foidart JM, Castronovo V and Green JR: Novel antiangiogenic effects of the bisphosphonate compound zoledronic acid. J Pharmacol Exp Ther 302: 1055-1061, 2002.
4. Fournier P, Boissier S, Filleur S, Guglielmi J, Cabon F, Colombel $\mathrm{M}$ and Clezardin P: Bisphosphonates inhibit angiogenesis in vitro and testosterone-stimulated vascular regrowth in the ventral prostate in castrated rats. Cancer Res 62: 6538-6544, 2002.

5. Giraudo E, Inoue M and Hanahan D: An amino-bisphosphonate targets MMP-9-expressing macrophages and angiogenesis to impair cervical carcinogenesis. J Clin Invest 114: 623-633, 2004.

6. Cetinkaya BO, Keles GC, Ayas B and Gurgor P: Effects of risedronate on alveolar bone loss and angiogenesis: a stereologic study in rats. J Periodontol 79: 1950-1961, 2008.

7. Meyer RD, Singh A, Majnoun F, Latz C, Lashkari K and Rahimi N: Substitution of C-terminus of VEGFR-2 with VEGFR-1 promotes VEGFR-1 activation and endothelial cell proliferation. Oncogene 23: 5523-5531, 2004.

8. Hiratsuka S, Minowa O, Kuno J, Noda T and Shibuya M: Flt-1 lacking the tyrosine kinase domain is sufficient for normal development and angiogenesis in mice. Proc Natl Acad Sci USA 95: 9349-9354, 1998.

9. Rahimi N, Dayanir V and Lashkari K: Receptor chimeras indicate that the vascular endothelial growth factor receptor-1 (VEGFR-1) modulates mitogenic activity of VEGFR-2 in endothelial cells. J Biol Chem 275: 16986-16992, 2000.

10. Skobe M, Rockwell P, Goldstein N, Vosseler S and Fusenig NE: Halting angiogenesis suppresses carcinoma cell invasion. Nat Med 3: 1222-1227, 1997.

11. Lindner V and Collins T: Expression of NF-kappaB and I kappaB-alpha by aortic endothelium in an arterial injury model. Am J Pathol 148: 427-438, 1996.

12. Dougher M and Terman BI: Autophosphorylation of KDR in the kinase domain is required for maximal VEGF-stimulated kinase activity and receptor internalization. Oncogene 18: 1619-1627, 1999.

13. Balla G, Vercellotti G, Eaton JW and Jacob HS: Heme uptake by endothelium synergizes polymorphonuclear granulocytemediated damage. Trans Assoc Am Physicians 103: 174-179, 1990.

14. Basi DL, Ross KF, Hodges JS and Herzberg MC: The modulation of tissue factor by endothelial cells during heat shock. J Biol Chem 278: 11065-11071, 2003.

15. Zhang ZQ, Notermans DW, Sedgewick G, et al: Kinetics of $\mathrm{CD}^{+} \mathrm{T}$ cell repopulation of lymphoid tissues after treatment of HIV-1 infection. Proc Natl Acad Sci USA 95: 1154-1159, 1998.

16. Guenther A, Gordon S, Tiemann M, Burger R, Bakker F, Green JR, Baum W, Roelofs AJ, Rogers MJ and Gramatzki M: The bisphosphonate zoledronic acid has antimyeloma activity in vivo by inhibition of protein prenylation. Int $\mathrm{J}$ Cancer 126: 239-246, 2010.

17. Clezardin P, Fournier P, Boissier S and Peyruchaud O: In vitro and in vivo antitumor effects of bisphosphonates. Curr Med Chem 10: 173-180, 2003.

18. Ewan LC, Jopling HM, Jia H, Mittar S, Bagherzadeh A, Howell GJ, Walker JH, Zachary IC and Ponnambalam S: Intrinsic tyrosine kinase activity is required for vascular endothelial growth factor receptor 2 ubiquitination, sorting and degradation in endothelial cells. Traffic 7: 1270-1282, 2006. 